\title{
Linee guida per lo sviluppo di modelli numerici di flusso in acquiferi porosi: Una proposta IRSA-ISPRA
}

\section{Guidelines for the development of numerical flow models in porous aquifers: An IRSA-ISPRA proposal}

\author{
Emanuele Romano - Istituto di Ricerca sulle Acque del Consiglio Nazionale delle Ricerche \\ Elisabetta Preziosi - Istituto di Ricerca sulle Acque del Consiglio Nazionale delle Ricerche \\ Federico Araneo - Servizio Geologico d'Italia - ISPRA - area GEO-PSC \\ Chiara Fiori - Servizio Geologico d'Italia - ISPRA - area GEO-PSC - email: chiara.fiori@isprambiente.it \\ Maurizio Guerra - Servizio Geologico d'Italia - ISPRA - area GEO-PSC
}

Keywords: Contaminated sites-conceptual model - data processing-flow model.

Parole chiave: Siti contaminati-modello concettuale-elaborazione dati-modello di flusso.

A livello nazionale, nell'ambito dei procedimenti di bonifica dei siti contaminati, disciplinati dalla parte Quarta - Titolo $\mathrm{V}$ del decreto legislativo 152 del 2006, l'implementazione di modelli matematici di flusso e di trasporto dei contaminanti è uno strumento che ha acquisito con il tempo sempre maggiore importanza, soprattutto ai fini della definizione e progettazione degli interventi di messa in sicurezza e bonifica delle acque di falda. Modelli matematici sono utilizzati anche a scala di bacino o sottobacino per la caratterizzazione e la gestione delle risorse idriche sotterranee.

In tale contesto risulta di primaria importanza che le amministrazioni pubbliche e gli organi di controllo, in qualità di revisori o committenti dei modelli stessi, si dotino di un riferimento metodologico che guidi da un lato lo sviluppo, dall'altro la valutazione dei modelli numerici.

Il documento ISPRA "Presentazione dei dati nell'elaborazione di modelli matematici di flusso", allegato (a partire dal 2013) ai pareri tecnici inerenti la valutazione dei modelli idrogeologici, ben lungi dal costituire una linea guida, aveva l'obiettivo di individuare i contenuti minimi necessari per poter valutare i modelli idrogeologici sviluppati a supporto della progettazione o della verifica degli interventi di bonifica/ messa in sicurezza delle acque sotterranee nei siti contaminati.

Un documento completo, redatto con lo scopo di fornire uno strumento operativo e di verifica di modelli numerici di flusso di acque sotterranee è stato sviluppato da IRSA-CNR per ARPA Umbria (IRSA-CNR, 2006); a nostra conoscenza, tale documento non è tuttavia mai stato utilizzato in ambito regionale.

Alla luce dell'utilizzo sempre più diffuso dello strumento di modellazione numerica nel campo idrogeologico, si è palesata l'opportunità di predisporre un riferimento operativo, sistematico e completo, utile al proponente e all'ente di controllo, anche nell'ottica di convergere verso un sistema di valutazione coerente a scala nazionale della validità di detti strumenti nel campo della caratterizzazione e bonifica dei siti contaminati.

A livello internazionale sono state sviluppate da diversi Enti "linee-guida" per lo sviluppo di modelli numerici di flusso e di trasporto in acquiferi porosi. Alcuni di essi si pongono l'obiettivo di fornire elementi di "best-practice", cioè di indicare la sequenza logica di operazioni necessarie affinché i risultati finali della modellazione siano matematicamente corretti e fisicamente rappresentativi e possano quindi essere considerati affidabili rispetto agli obiettivi specifici dichiarati (Boonstra and de Ridder, 1981; Hill et al. 2000; MurrayDarling Basin Commission, 2001; Willing, 2007; Rassam et al. 2012; Trevor et al., 2016). Altri si pongono l'obiettivo di fornire elementi di valutazione dei modelli stessi a partire da analisi di best-practice (Reilly e Harbaugh, 2004; Merrick et al, 2002; Middlemis, 2004; Barnett et al. 2012; Wels et al., 2012). Secondo Reilly e Harbaugh (2004) la difficoltà principale che gli organi di controllo e, più in generale, gli utenti finali, si trovano ad affrontare nell'utilizzo o nella valutazione di prodotti di tipo modellistico, è l'analisi oggettiva dei punti di forza e di debolezza dei modelli stessi senza la necessità di riprodurre l'intera analisi. Indipendentemente dall'obiettivo finale e dall'indirizzo generale, tutte le linee-guida citate individuano alcuni elementi imprescindibili, in mancanza dei quali la validità del prodotto finale e dunque la sua utilizzabilità operativa sono messi in dubbio.

Basandoci su analoghi diagrammi proposti in letteratura (i.e. Murray-Darling Basin Commission, 2001; Anderson e Woessner, 1992; Spitz e Moreno, 1996; Barnett et al. 2012; Alaska Department of Environmental Conservation, 2017) le Linee Guida da noi proposte sono basate sullo schema operativo presentato in figura 1 . Esso è stato sviluppato specificamente per modelli di flusso di acque sotterranee, ma può essere applicato sostanzialmente con la stessa struttura anche a modelli di trasporto.

In sostanza gli elementi fondamentali su cui si deve impostare un modello numerico affidabile sono costituiti dai seguenti passi:

1. Definizione degli obiettivi del modello. Questa fase prevede l'individuazione del fenomeno che si intende modellare, delle variabili di input, delle variabili di output e dei parametri necessari per lo sviluppo del modello di flusso.

2. Analisi dei dati disponibili. Obiettivo di questa fase è la raccolta e l'organizzazione dei dati sperimentali disponibili e la valutazione della loro adeguatezza rispetto agli obiettivi del modello individuati nel punto 1. 


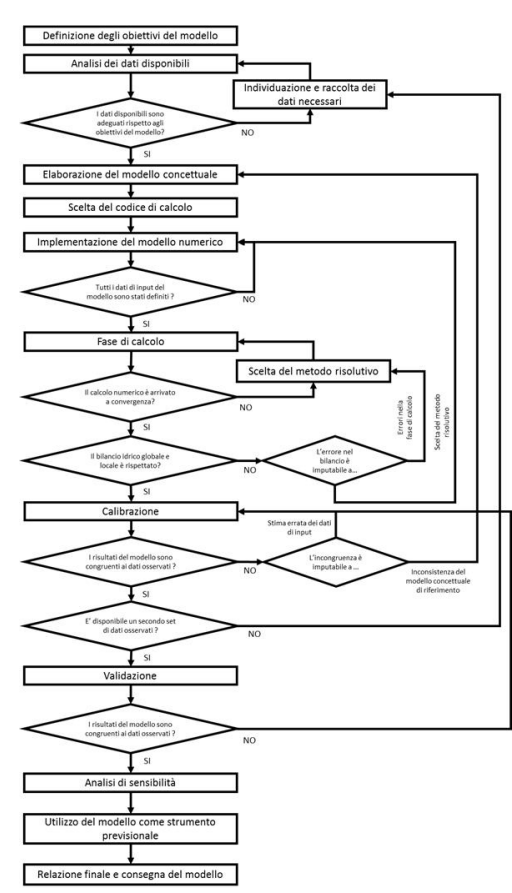

Fig. 1: Diagramma di flusso relativo all'implementazione di un modello numerico.

Fig. 1: Flow chart related to a numerical model processing .

3. Elaborazione del modello concettuale di riferimento. Le caratteristiche geometriche dell'acquifero, i dati relativi alle variabili di input/output e ai parametri fenomenologici vengono unificati in un unico schema interpretativo che descrive le caratteristiche del flusso idrico sotterraneo alla scala scelta.

4. Calibrazione del modello. Nel corso di questa fase si cercano, mediante opportune metodologie, i valori ottimali dei parametri e/o delle variabili di input tali che la condizione di flusso modellata sia il più vicina possibile alla condizione di flusso osservata.

5. Validazione del modello. La fase di validazione del modello consiste nell'utilizzare il modello calibrato per simulare una situazione di flusso differente da quella utilizzata in fase di calibrazione. Se la validazione non dà risultati soddisfacenti può essere necessaria una revisione sia della fase di calibrazione, sia della fase di elaborazione del modello concettuale.

6. Utilizzo del modello come strumento previsionale. In molti casi la "previsione" delle variazioni del campo di moto e della quantità di acqua immagazzinata o rilasciata indotte da variazioni degli input costituisce il fine ultimo della modellazione.

7. Individuazione dei criteri di reporting. Le modalità di presentazione dei risultati devono consentire di individuare gli input e tracciare tutto il processo decisionale (es. inclusione nel report finale dei files necessari alla replica delle simulazioni e ad una eventuale implementazione successiva del modello).

\section{Modellistica e Idrogeologia}

I modelli possono essere definiti come rappresentazioni matematiche del comportamento fisico di un sistema reale.

Facendo riferimento ad un approccio di tipo deterministico un sistema fisico, anche complesso, può essere rappresentato come relazione causa-effetto tra un insieme di fenomeni supposti "causali" (le "forzanti") rappresentati da variabili indipendenti (o variabili di input) e fenomeni che da essi sono determinati, rappresentati da variabili dipendenti (o variabili di output). Tale relazione è espressa mediante equazioni matematiche nelle quali compaiono, oltre alle variabili di input e di output, i parametri fenomenologici, che nel caso della modellazione del flusso nel mezzo poroso sono costituiti dai parametri idrogeologici conducibilità idraulica k e coefficiente di immagazzinamento s.

I fenomeni naturali sono sempre caratterizzati da un elevato grado di complessità che si manifesta sia nell'identificazione dei processi fisici che concorrono a determinare il fenomeno osservato, sia nella descrizione dei fenomeni stessi, cioè nel tentativo di descrivere in termini qualitativi e quantitativi ciò che si osserva.

Quando si analizzano i sistemi naturali spesso non è possibile, né utile, descrivere nel dettaglio tutti i fenomeni coinvolti, ma risulta necessario individuare gli elementi che si considerano significativi. Conseguenza di ciò è che non può esistere un modello universalmente adeguato alla descrizione di un sistema, ma la bontà di un modello è valutabile esclusivamente in relazione alla capacità di rappresentare una certa realtà fisica a priori individuata.

In altre parole lo sviluppo di un modello matematico ha come punto di partenza l'individuazione degli obiettivi del modello stesso, e quindi dei fenomeni che si vogliono rappresentare. Tale rappresentazione consta essenzialmente di tre elementi:

1. le variabili di output considerate rappresentative del fenomeno che si intende modellare: la simulazione dei dati osservati relativi alle variabili di output è l'obiettivo primario di un modello matematico;

2. le variabili di input, che si ritiene concorrano al realizzarsi del fenomeno osservato;

3. la relazione, espressa mediante equazioni matematiche, che lega le variabili di input con le variabili di output attraverso i parametri idrodinamici.

I modelli matematici di tipo idrogeologico possono essere inquadrati nello schema prima presentato.

Appare evidente come, in tale approccio, sia di fondamentale importanza la valutazione dell'adeguatezza dei dati elaborati ed inseriti nello sviluppo di un modello.

Ciò significa far riferimento ad uno schema operativo nel quale si faccia precedere all'analisi dei dati osservati disponibili, un'analisi a priori volta a identificare quali siano i dati necessari affinché il processo che si intende modellare sia adeguatamente descritto, secondo lo schema di Figura 3. 


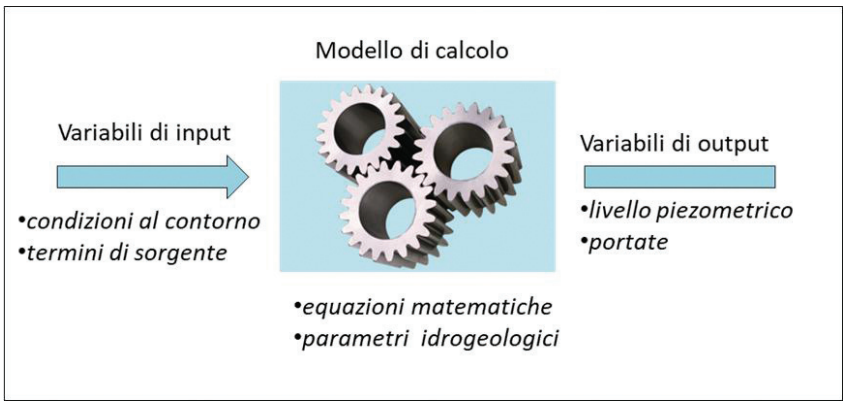

Fig. 2: Sviluppo di un modello matematico con riferimento all'ambito idrogeologico.

Fig. 2: Development of a mathematical model with reference to the hydrogeological framwork.

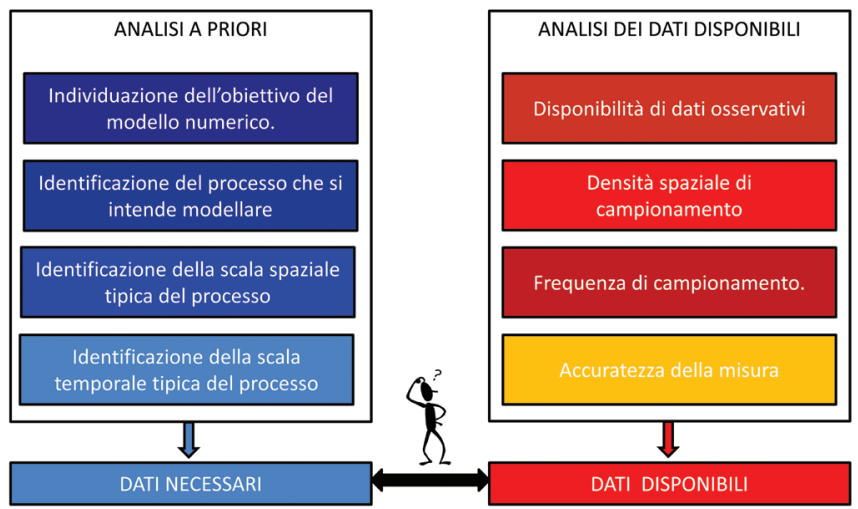

Fig. 3: Diagramma di flusso per la valutazione della rappresentatività dei dati osservati disponibili.

Fig. 3: Flow chart for the evaluation of observed data.

Solo successivamente a tale fase potrà essere definito il modello concettuale idrogeologico di riferimento che il modello numerico tradurrà in termini quantitativi, mettendo in relazione l'insieme dei dati a disposizione mediante equazioni della fisica matematica.

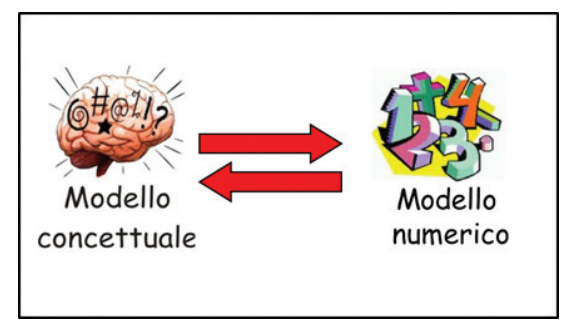

Fig. 4: Il modello concettuale ed il modello numerico devono interfacciars iterativamente.

Fig. 4: Conceptual model and numerical iteratively dialogue.

Tale percorso metodologico, unitamente agli altri step tecnici di cui sopra, è stato strutturato ed illustrato nelle "Linee guida per lo sviluppo di modelli numerici di flusso in acquiferi porosi" redatte da IRSA-CNR ed ISPRA con l'obiettivo primario di fornire un pratico supporto agli operatori pubblici e privati del campo delle bonifiche nei siti contaminati.

Le linee guida sono già state presentate, all'interno del volume monografico SIGEA "Bonifica dei siti inquinati", all'even- to REMTECH del 19 settembre 2019 (Romano et al. 2019).

Lulteriore e necessario passo in avanti, ai fini della fruizione finale di tale prodotto tecnico, sarà quello di considerare la possibilità di condividere questo percorso nell'ambito del SNPA anche al fine di affrontare criticità specifiche derivanti dall'esperienza maturata dalle singole agenzie.

\section{BIBLIOGRAFIA}

Alaska Department of Environmental Conservation (2017) Fate and Transport Modeling Guidance.

Anderson MP and Woessner WW (1992) Applied Groundwater Modeling, Academic Press, Inc., S. Diego

Barnett et al. (2012) Australian groundwater modelling guidelines, Waterlines report n. 82, National Water Commission, Canberra.

Boonstra J and de Ridder NA (1981) Numerical modelling of ground water basins - A user oriented manual. IILRI, P.O. Box. 6700A Wageningen, The Netherlands, Publication No. 29, p. 226.

Hill MC, D'Agnese FA, Faunt CC (2000) Guidelines for model calibration and application to flow simulation in the Death Valley regional groundwater system. Calibration and Reliability in Groundwater Modelling (Proceedings of the ModelCARE 99 Conference held at Zurich, Switzerland, September 1999). IAHS Publ. no. 265.

IRSA-CNR (2006) Linee Guida per lo sviluppo di modelli numerici di flusso in ambito idrogeologico. In "Convenzione per la formazione, ricerca e supporto metodologico nel monitoraggio quantitativo delle acque sotterranee mediante implementazione di modellistica idrogeologica tra ARPA Umbria e IRSA-CNR"

Merrick NP, Middlemis H, Ross JB (2002) Groundwater Modelling Guidelines for Australia - recommended Procedures for Modelling Reviews. International Groundwater Conference. Balancing the Groundwater Budget. Northern Territory. Australia

Middlemis H (2004) Benchmarking best practice for groundwater flow modelling. Kent Town, South Australia, Australia.

Murray-Darling Basin Commission (MDBC) (2001) Groundwater flow modelling guideline, report prepared by Aquaterra.

Rassam DW, Jolly I, Pickett T (eWater Cooperative Research Center) (2012) Guidelines for modelling groundwater-surface water interaction in eWater source: towards best practice model applications. ISBN: 978-1-921543-59-3

Romano E, Baldi D, Araneo F, Fiori C, Guerra M (2019) Il contributo della modellistica numerica nella gestione dei siti contaminati: una proposta di linee guida operative. In "Bonifica dei siti inquinati", supplemento n.2/209, Ed. Daniele Baldi,

Reilly TE and Harbaugh AW (2004) Guidelines for evaluating groundwater flow models, US Geological Survey, Scientific Investigations Report 2004-5038.

Trevor J, Godwin T, McManus D, Nordberg M, Shannon H, Springhorn S (2016) Modeling Best Management Practice. California Department of Water Resources.

Wels C, Mackie D, Scibek J (2012) Guidelines for Groundwater Modelling to Assess Impacts of Proposed Natural Resource Development Activities. Report no. 194001. Ministry of Environment - Water Protection \& Sustainability Branch.

Willing P. (2007). A Nontechnical Guide to Groundwater Modeling. Natural Resources Defense Council. 36 pp. 